\title{
LA ECONOMÍA INSTITUCIONAL Y SU RELACIÓN CON LOS SECTORES PRODUCTIVOS
}

\author{
INSTITUTIONAL ECONOMICS AND ITS RELATIONSHIP WITH PRODUCTIVE SECTORS
}

\section{A ECONOMIA INSTITUCIONAL E SUA RELAÇÃO COM OS SETORES PRODUTIVOS}

\section{María Leonela Artavia Jiménez ${ }^{1}$ \\ Shirley Benavides Vindas ${ }^{2}$ \\ Álvaro Martín Parada Gómez ${ }^{3}$}

\begin{abstract}
Resumen
El artículo aborda la teoría de la economía institucional desde la perspectiva de su relación con los sectores productivos. El objetivo es dilucidar los principales aportes de la teoría institucionalista en materia de formulación de políticas de apoyo a los sectores productivos de países en desarrollo. Se aborda el rol del Estado y su papel regulador, los bienes públicos y la importancia de las instituciones en el ordenamiento socioeconómico del país. Del análisis realizado surge la conclusión de que un mayor desarrollo institucional dirigido en dos líneas, la primera hacia el fomento del desarrollo integral de las personas, que son quienes conforman la sociedad e integran la fuerza laboral, y la segunda, hacia la promoción de dinámicas innovadoras que incluyan la implementación de nuevas tecnologías, es una de las rutas para el fortalecimiento del desarrollo productivo de los países en desarrollo.
\end{abstract}

Doi: http://dx.doi.org/10.15359/eys.23-53.5

Fecha de recepción: 26-01-2018. Fechas de reenvíos: 29-01-2018, 26-02-2018, 26-04-2018, 28-05-2018, 8-06-2018. Aceptado el 10-06-2018. Publicado el 15-06-2018.

${ }^{1}$ Licenciada en Economía. Académica e investigadora del Programa Sectores Productivos, Competitividad y Desarrollo de la Escuela de Economía, Universidad Nacional, Costa Rica. Correo electrónico: leonela.artavia.jimenez@una.ac.cr

${ }^{2} \mathrm{PhD}$. Académica e investigadora del Programa Sectores Productivos, Competitividad y Desarrollo de la Escuela de Economía, Universidad Nacional, Costa Rica. Correo electrónico: shirley.benavides.vindas@una.ac.cr

${ }^{3} \mathrm{PhD}$ en Economía. Académico e investigador del Programa Sectores Productivos, Competitividad y Desarrollo, Escuela de Economía, Universidad Nacional, Costa Rica. Correo electrónico: agomez@una.ac.cr 84

María Leonela Artavia Jiménez, Shirley Benavides Vindas y Álvaro Martín Parada Gómez

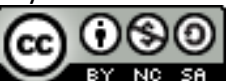

Revista Economía y Sociedad by Universidad Nacional is licensed under a CreativeCommons Reconocimiento-NoComercial- 
Palabras claves: economía institucional; desarrollo; sectores productivos; políticas públicas; regulación

\begin{abstract}
Institutional economics theory regarding its relationship with productive sectors is discussed in this article. The main objective of the paper is to clarify the main contributions of institutionalism theory in the formulation of policies to support the productive sectors of developing countries. To this end, the article discusses the role of the State as a regulatory entity, public goods, and the importance of institutions in the socio-economic order of the country. From this analysis, it is concluded that one of the routes to strengthen the productive development of developing countries is having greater institutional development for two purposes: first towards the promotion of the integral development of people, who make up the society and are part of the workforce, and second towards the promotion of innovative dynamics that would include the implementation of new technologies.
\end{abstract}

Keywords: institutional economics; development; productive sectors; public policies; regulation

\title{
Resumo
}

O artigo aborda a teoria da economia institucional desde a perspectiva de sua relação com os setores produtivos. O objetivo é elucidar as principais contribuições da teoria institucionalista em matéria de formulação de políticas de apoio aos setores produtivos dos países em desenvolvimento. Aborda-se o papel do Estado e seu papel regulador, os bens públicos e a importância das instituições na ordem socioeconômica do país. A partir da análise realizada, conclui-se que um maior desenvolvimento institucional é direcionado em duas linhas, a primeira voltada para a promoção do desenvolvimento integral das pessoas, que compõem a sociedade e integram a força de trabalho, e a segunda, para a promoção de dinâmicas inovadoras que incluam a implementação de novas tecnologias, sendo um dos caminhos para o fortalecimento do desenvolvimento produtivo dos países em desenvolvimento.

Palavras-chave: Economia institucional; desenvolvimento; setores produtivos; políticas públicas, regulamentação. 


\section{Introducción}

El desarrollo productivo en países como Costa Rica se ha ido dando en medio de un pulso entre la intervención del Estado y el libre mercado, este último promovido con mayor fuerza desde la década de los 80. Esto ha dado pie a una gran cantidad de posiciones sobre el tema, desde la perspectiva liberal se defiende que sean las fuerzas del mercado las que definan la senda del desarrollo, mientras que otros defienden la intervención del Estado y su papel de supervisor y regulador del mercado. De manera que este asunto entraña no solamente un tema desde el orden de lo ideológico, sino también de lo institucional; es decir, desde las reglas que demarcan el proceder de los individuos y su conjunto como sociedad (North, 1981).

En virtud de ello, se toma en este artículo la teoría institucionalista, que se agrupa en dos vertientes conocidas como la Economía Institucional Original (EIO) y la Nueva Economía Institucional (NEI), ambas útiles en la orientación de la política pública y sectorial desarrollada por los distintos gobiernos y Estados Nacionales, para dar respuesta a un par de interrogantes fundamentales relacionadas con el tema de los sectores productivos: ¿cuál es la contribución del enfoque institucionalista para el mejoramiento de políticas de apoyo al desarrollo productivo de países del sur global? y ¿cuál es, teóricamente, la función del mercado y del Estado en el desarrollo productivo desde el enfoque institucionalista? En el afán de responder a estas cuestiones, se establece como propósito explicar los fundamentos de este enfoque en relación con el desarrollo productivo y la competitividad empresarial, así como ofrecer un debate teórico relacionando la dinámica de los sectores productivos costarricenses desde la economía institucional, a partir de la revisión de las teorías de la EIO y la NEl y de la experiencia del equipo investigador en el análisis de dichos sectores.

El texto consta de nueve secciones. En la primera de ellas se aborda el tema del desarrollo productivo en el contexto del institucionalismo; en la segunda, la concepción de la institucionalidad; en la tercera, se explica el cambio institucional como proceso, para lo cual se incorpora la diferenciación entre organizaciones e instituciones, así como la importancia de la cultura; en el cuarto segmento se habla de las instituciones políticas; para luego, en la quinta sección, tocar el tema del Estado. Además, en la sexta se enuncia el tema de los costos de transacción y su importancia dentro de la teoría económica; en la sétima, la relación de la institucionalidad con el desarrollo; y en la última parte se expone el tema de la regulación dentro de la teoría institucionalista. Se cierra con un apartado de conclusiones.

\section{El desarrollo productivo en el contexto del institucionalismo}

Para el análisis de los sectores productivos no solo se debe tomar en cuenta aspectos macro y microeconómicos o elementos que posibilitan la competitividad; según Esser, Hillebrand, Messner \& Meyer-Stamer (1996), debe valorarse todo lo relacionado con el nivel 86

María Leonela Artavia Jiménez, Shirley Benavides Vindas y Álvaro Martín Parada Gómez

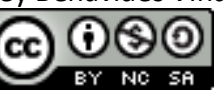

Revista Economía y Sociedad by Universidad Nacional is licensed under a CreativeCommons Reconocimiento-NoComercial- 
mesoeconómico, el cual formula, gestiona y ejecuta las políticas públicas que se generan para mejorar las condiciones de funcionamiento del mercado, dadas las imperfecciones que limitan la competitividad. En este nivel, las instituciones $y$, particularmente, su eficiencia, se constituyen como un criterio de óptimo funcionamiento, debido al rol mediante el cual se presentan las reglas de juego que rige el comportamiento del mercado, en el entorno exógeno de los sectores productivos que les posibilita o limita su desarrollo.

Aunado a lo anterior, el territorio se constituye como otro elemento a considerar en la valoración de la eficiencia del nivel meso, y de él sus condiciones diferenciadoras, que son expresiones del tipo de desarrollo que han seguido, erigiéndose centros de desarrollo y regiones periféricas. En esta consideración, las organizaciones -públicas y privadas- deben tomar en cuenta las diferencias que se presentan en ellos, para responder adecuadamente a sus requerimientos específicos. Asimismo, desde la institucionalidad se puede estimular los sectores productivos mediante la articulación adecuada de los mercados de trabajo y el impulso a la educación, salud, seguridad, sector financiero y de transportes, así como a organizaciones gremiales y empresariales que, en conjunto, pueden aportar al desarrollo sostenible en cada territorio, según sus particularidades.

Por otra parte, las instituciones importan, con base en estas se establece vincularse con las relaciones internacionales, ya que organizan y regulan los flujos comerciales en el mercado internacional, así como los flujos de inversión extranjera directa. En una escala global en la que hay estándares de intercambio muy elevados, la institucionalidad favorece o no esos enclaves; un ejemplo de ello es la gestión gubernamental de las exportaciones, a través de la cual se facilitan vínculos empresariales, educativos, tecnológicos y burocráticos que articulan los mercados de bienes y servicios y de trabajo.

Como pensamiento, la economía institucional presenta tal variedad que se hace preciso aglutinarlo en dos grandes bloques: el primero es el llamado Economía Institucional Original (EIO) y el segundo corresponde a la Nueva Economía Institucional (NEI). De esta última, North (1981, 1990, 1994) es el mayor exponente, considerado economista histórico, cuyos aportes a esta rama de pensamiento le hicieron acreedor del Premio Nobel de Economía en el año 1993.

\section{Hacia una concepción de la institucionalidad}

\subsection{Las instituciones}

El sistema económico integrado por empresas, familias, bancos y gobierno, es dinamizado por la institucionalidad pública; estas generan las reglas del juego en una sociedad o, más formalmente, son las limitaciones ideadas por el ser humano que dan forma a la interacción

María Leonela Artavia Jiménez, Shirley Benavides Vindas y Álvaro Martín Parada Gómez 
humana (North, 1990). Estas reglas influyen necesariamente en la evolución del desempeño de las economías y se hace relevante determinar qué es lo que genera el cambio institucional (ver apartado 3), para entender de una mejor manera qué puede estar influenciando el desempeño económico. Típicamente, en los gobiernos se operacionalizan políticas, medidas y directrices que establecen las reglas de juego en las distintas dimensiones de intervención, tanto en lo público como en lo privado.

Por otra parte, las instituciones cumplen una función de reducción de la incertidumbre, pues les ofrecen a las personas una estructura estable de lo que deben o no hacer ante diferentes situaciones de la vida, de acuerdo con el lugar donde se encuentren. La estabilidad es relativa, porque en el sistema económico surgen fuerzas económicas, políticas y sociales que introducen desequilibrios en la institucionalidad. En la conceptualización de la institucionalidad se incorporan elementos que la teoría neoclásica no ha integrado en su análisis, como lo es la valoración de las externalidades, los costos de transacción, la presencia de la regulación, entre otros. Adicionalmente, factores relacionados con el lugar donde habitan las personas que integra aspectos como el idioma, la cultura y un determinado esquema de valores. Estas variables condicionan la institucionalidad de cada región en un proceso de retroalimentación dado que las instituciones "definen y limitan el conjunto de elecciones de los individuos" (North, 1990 , p. 4).

La economía institucional ofrece un marco más amplio de análisis en comparación con la propuesta tradicional de la economía neoclásica porque integra el proceso económico, social, ambiental y cultural como parte de ese sistema. Desde la perspectiva del desarrollo productivo, las relaciones sociales y económicas son complejas y los precios explican parcialmente la dinámica de los agentes económicos; por ejemplo, los productores ante desequilibrios en los mercados requieren apoyo institucional para mantener su buen posicionamiento en los mercados, incluso los gobiernos mantienen subsidios en productos muy específicos como café y arroz, en países en desarrollo como Costa Rica.

\subsection{Sectores productivos y la dinámica de las instituciones en Costa Rica}

La dinámica institucional es fundamental para los sectores productivos, pues dependiendo de su conformación y la intencionalidad de estas se impulsará el desarrollo o se contendrá. El Estado, como ente encargado de articular políticas públicas se encarga del diseño e implementación de las mismas, esto muchas veces en coordinación con organizaciones privadas. En el caso costarricense, gran parte de esta responsabilidad recae sobre el Ministerio de Economía, Industria y Comercio de Costa Rica (MEIC), mediante su Consejo de Promoción de la Competencia (COPROCOM), el cual tiene como propósito:

88

María Leonela Artavia Jiménez, Shirley Benavides Vindas y Álvaro Martín Parada Gómez

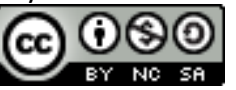

Revista Economía y Sociedad by Universidad Nacional is licensed under a CreativeCommons Reconocimiento-NoComercial- 
La tutela y la promoción del proceso de competencia y libre concurrencia, investigando y sancionando las prácticas monopolísticas y otras restricciones al funcionamiento eficiente del mercado. Somos un órgano de desconcentración máxima adscrito al Ministerio de Economía, Industria y Comercio.

En un mercado con pocos participantes las empresas se ponen de acuerdo y funcionan como un monopolio. Nuestro objetivo es evitar que las empresas realicen conductas que les permitan comportarse como un monopolio por medio del cumplimiento de la Ley de Promoción de la Competencia (Ley $\mathrm{N}^{\circ}$ 7472). (COPROCOM, 2017, párrafos 1,2.)

Por otra parte, el Estado suele ejercer la función de ente regulador, que consiste en generar las condiciones necesarias para el buen desempeño competitivo de las empresas de manera simétrica. Respecto a os sectores productivos, le corresponde al MEIC ejercer dicha función, para lo cual se han propuesto los siguientes objetivos:

- Fortalecer la competitividad del sector productivo nacional, mediante la mejora regulatoria y la simplificación de trámites claves.

- Facilitar condiciones que permitan el acceso y el adecuado funcionamiento del mercado nacional, que favorezcan el desarrollo integral y equitativo del país.

- Promover un marco regulatorio claro y eficiente que brinde seguridad jurídica al administrado y propicie servicios del Estado eficientes. (MEIC, 2017).

Esta labor del MEIC da indicios de que existe una estructura institucional en el país la cual busca el buen desenvolvimiento de los sectores productivos; todo ello con apoyo a temas estratégicos tales como procesos de innovación, de conocimiento y aprensión de las nuevas tecnologías desarrolladas, que generan mejores condiciones para la producción y comercialización del parque empresarial de los diversos territorios. En este sentido, Caselet (2004) señala que:

Las trayectorias institucionales generadas y readaptadas por la acción de los actores institucionales (empresas, institutos de investigación, organizaciones intermedias) construyen el conocimiento específico y los procesos que este implica. Las instituciones se transforman frente a las nuevas exigencias de la producción y del contexto internacional y local. En algunos casos, las modificaciones llevan a una reestructuración de metas y programas para atender demandas específicas. En otros, se crean nuevas instituciones más flexibles para responder a las necesidades de la producción y del conocimiento no resueltas por las instituciones existentes [...] La naturaleza de la interacción basada en la concentración de los apoyos públicos y privados para activar la modernización tecnológica y el fomento productivo plasmó un cambio en el contexto institucional. (p. 180) 
Desde la anterior perspectiva, las redes institucionales que además están integradas por ministerios como el de trabajo (MTSS), el de Agricultura y Ganadería (MAG), Comercio Exterior (COMEX) y la Promotora de Comercio Exterior (PROCOMER), en conjunto con la labor de los municipios, significan una base importante para el desempeño de las empresas, debido a que generan o limitan las condiciones para lograr una adecuada competitividad de los sectores productivos y del país en términos generales. Además, desde entidades como el Instituto Nacional de Aprendizaje (INA), las universidades públicas y privadas, o la labor del Ministerio de Educación Pública (MEP), se refuerza el sector productivo a través de los mercados de trabajo. Esta labor es de gran importancia en términos de su potencial de aportar al nivel de especialización de las personas, aspecto que implica una mejor interacción de la oferta y demanda del mercado laboral, lo cual es relevante en función de reducir el desempleo por desequilibrios entre la demanda de empleo y el perfil ofrecido por los trabajadores.

\section{Proceso de cambio institucional}

\subsection{Instituciones y organizaciones}

La distinción entre organizaciones e instituciones se relaciona con el rol de las reglas del juego y de los jugadores. En este sentido, North (1981) afirma que las organizaciones son "grupos de individuos limitados por algún propósito común para lograr objetivos políticos, sociales, económicos y educativos" (p. 5). El progreso económico es impulsado por la institucionalidad, lo cual ocasiona que unas sociedades sean más desarrolladas que otras; es decir, que unas de ellas permanezcan estancadas por décadas mientras otras avanzan con mayor dinamismo. Esta capacidad de generar desarrollo por parte de las instituciones dependerá de la posibilidad de irse renovando el tiempo para adaptarse a los requerimientos cambiantes de las organizaciones; esto es lo que recibe el nombre de cambio institucional, el cual, según North (1981), condiciona la evolución del desempeño de las economías.

El cambio institucional tiene implicaciones económicas y sociales, porque las instituciones determinan las organizaciones y, al mismo tiempo, esas organizaciones afectan la evolución de las instituciones, generándose un proceso de impulso en doble vía: institucionesorganizaciones. Desde NEI, las instituciones, junto con las limitaciones ordinarias de la teoría económica, determinan las oportunidades que hay en una sociedad y que las organizaciones pueden aprovechar. No se cuestiona el funcionamiento de las instituciones estrictamente, el cual es ampliamente criticado por la presencia de burocracia, exceso de intervención del Estado, regulación excesiva, ineficiencia en los servicios que ofrece, entre otros, pero este tema puede entenderse como un mal desempeño institucional.

La interacción entre las organizaciones, ya sean i. políticas: partidos políticos, senado, concejo municipal, cuerpos reguladores; ii. económicas: empresas, sindicatos, granjas familiares, 90

María Leonela Artavia Jiménez, Shirley Benavides Vindas y Álvaro Martín Parada Gómez

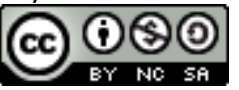

Revista Economía y Sociedad by Universidad Nacional is licensed under a CreativeCommons Reconocimiento-NoComercial- 
cooperativas; iii. sociales: iglesias, clubes, asociaciones deportivas; iv. educativas: colegios, universidades, centros vocacionales) y las reglas de juego establecidas (instituciones) en cada sociedad determinarán su nivel de progreso económico. En este sentido, al estar las organizaciones conformadas por grupos de individuos aglutinados con el propósito de alcanzar ciertos objetivos, se verán en la necesidad de demandar individuos que posean conocimientos acordes con las actividades necesarias para alcanzarlo, estas destrezas demandadas por las organizaciones condicionan la evolución y el uso del conocimiento de las personas. (North, 1990), un ejemplo de ello es la prospección laboral, un estudio de este tipo indica cuales son las necesidades en términos de perfil de los trabajadores que requieren, de manera que en función de ello se ofertará la profesionalización y la capacitación, de manera que no hayan distorsiones entre la oferta y la demanda de trabajo en los territorios. Sin embargo, la institucionalidad puede y debe ir más allá y dirigirse hacia mejoras en la educación de las personas y la búsqueda de la ampliación del acceso a esta, con dos fines concretos: el primero de ellos, referido a la ampliación de las libertades de las personas que se logran con mejores niveles de instrucción, tanto en la toma de decisiones como en la movilidad social que esta les otorga, y la segunda, relacionada con el fortalecimiento de los mercados laborales, por la mejora en temas de productividad y de mejor calidad de empleo asociada a mayores niveles de escolaridad.

En este sentido, partiendo de que las reglas de juego se establecen a través de leyes, normas y directrices las cuales regulan la institucionalidad con el fin de alcanzar los objetivos que pretenden la solución de los problemas y necesidades planteadas por la sociedad, la pregunta que surge es si este conjunto de reglas es el adecuado para que las organizaciones contribuyan al desarrollo productivo y al mejoramiento del disfrute de la vida de las sociedades en general.

En relación con los sectores productivos, en el caso costarricense, se encuentra una serie de instituciones que son las responsables de facilitar el diseño y realizar la ejecución de la política pública que proporciona las condiciones para una mejor plataforma de la competitividad del país y de las regiones y de los sectores de manera particular. EI MEIC, MAG, COMEX, MTSS, MEP, los ministerios de Salud (MS), de Seguridad Pública (MSP) y de Ciencia Tecnología y Telecomunicaciones (MICIIT) se convierten básicamente en una red, desde la perspectiva del Gobierno Central, que debe estar en constante coordinación para generar las condiciones necesarias para el desarrollo de los sectores productivos; esto es, el cambio institucional requerido para mejorar el desenvolvimiento del país.

Por su parte, dentro de la administración pública las instituciones descentralizadas que deben unirse a dicho esfuerzo se encuentran al menos los siguientes institutos: Nacional de Aprendizaje (INA), de Seguros (INS), de Desarrollo Rural (INDER), de Fomento a las Cooperativas (INFOCOOP), el Costarricense de Ferrocarriles (INCOFER), Acueductos y Alcantarillados (AyA), Costarricense de Electricidad (ICE), así como la red de universidades e institutos de investigación y el sistema bancario. Todos ellos operacionalizan condiciones esenciales para fortalecer $y$ 
ejecutar la política pública por el adecuado desarrollo del país y, por ende, a la plataforma que la empresariedad requiere para su positivo desempeño a través del mercado laboral y su valor agregado a la producción interna.

A partir de la comprensión de la interacción entre instituciones y organizaciones, cobra importancia la incorporación de un elemento vital dentro de la economía institucional, al momento de analizar el proceso de cambio institucional: el proceso de aprendizaje. Esto porque, según North (1994), el cambio en el largo plazo es impulsado fundamentalmente por el aprendizaje de individuos y empresarios de las organizaciones. La incorporación de este elemento es un punto de quiebre importante con respecto a la teoría neoclásica, pues rompe con el supuesto de racionalidad de que al maximizar el beneficio individual se maximiza el colectivo, insertando la variable aprendizaje como fuente de desarrollo colectivo (de las organizaciones) y, con ellas, de la sociedad. A este respecto, Robles (1998) indica que North "propone desechar la hipótesis de racionalidad que sustenta a la teoría económica convencional y explorar constructivamente la naturaleza del aprendizaje humano" (p. 15).

Todos los esfuerzos institucionales e incluso organizacionales deben generar una articulación estratégica que posibilite el acceso oportuno a las fuentes de conocimiento y de innovación, así como de experiencias para el desarrollo productivo, no solo dirigido al actual y consolidado parque empresarial sino también al incipiente, como lo son los emprendimientos estimulados por procesos de formación e incubación que ofrece un país o región; esto con el propósito de evitar la heterogeneidad en las condiciones de mercado, especialmente para los más sensibles a cambios bruscos, así como los micro y pequeños empresarios, que aún no cuentan con una organización empresarial consolidada que les dé la línea base para su adecuado funcionamiento, en el corto y largo plazo, y que facilite su inserción y sostenibilidad en los mercados internacionales.

En resumen, una adecuada red de instituciones, desarrolladas sobre la base de un proceso sólido de aprendizaje que impulse su cambio en respuesta a los requerimientos de las organizaciones y de los sectores en general, conducirá a la generación de condiciones para un mejor desenvolvimiento de los sectores productivos, que respondan oportunamente a las fallas de mercado.

\section{Instituciones políticas}

La NEl establece que las instituciones políticas y económicas tienen en sus manos el comportamiento del desempeño económico; de acuerdo con la manera en que estas instituciones se desenvuelvan en cada sociedad, así será el buen o mal desempeño económico. Para North (1994), las instituciones forman la estructura de incentivos de los individuos que interactúan en sociedad, por consiguiente, las instituciones políticas y económicas son los 92

María Leonela Artavia Jiménez, Shirley Benavides Vindas y Álvaro Martín Parada Gómez

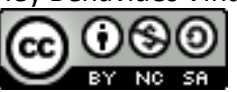

Revista Economía y Sociedad by Universidad Nacional is licensed under a CreativeCommons Reconocimiento-NoComercial- 
determinantes fundamentales del desempeño económico en el largo plazo. Las elecciones que hacen los individuos dependen del aprendizaje acumulativo, que se transmite culturalmente de generación en generación.

Es importante considerar que factores como el conocimiento y la tecnología tienen un lugar dentro del marco analítico de la NEI, como medios para alcanzar los límites superiores de bienestar para los seres humanos; sin embargo, es la institucionalidad económica y política la que determina tanto el desarrollo de un conocimiento elevado como el tipo de tecnología empleada, por lo cual, determinan el desempeño económico y el crecimiento del acervo de conocimiento, como se explicó anteriormente. En este sentido, el grado de cooperación (argumento contrario al supuesto de racionalidad) y competencia, desarrollado entre las personas, así como los sistemas de aplicación de las reglas (formales e informales) que organizan la actividad humana son vitales para entender el cambio económico. Precisamente, esas reglas permiten comprender el sistema que limita o incentiva y moldean la actividad económica de los sectores productivos; además de esto, determinan la distribución de riqueza e ingresos.

Si se incluye en el análisis institucional a los procesos productivos, fácilmente se encuentra el camino para llegar a la comprensión del desempeño económico y las interrelaciones entre los dos aspectos citados como mecanismo de dinamizar o limitar el desarrollo. La teoría neoclásica ha sido una ruta inadecuada para el análisis y la determinación de las políticas públicas que induzcan el desarrollo. Asimismo, se orientó al funcionamiento de los mercados, pero no necesariamente como éstos se desenvuelven, lo que limita su comprensión a través del mejoramiento de su desempeño, debido a que mediante la interacción de las instituciones, organizaciones y empresas, conforman la evolución institucional en una economía, lo que puede llevar a una metáfora en la cual las instituciones son el marco que define las reglas del juego, las organizaciones y sus empresarios son los jugadores y, dependiendo de la calidad y oportunidad de la interacción de todos ellos, surge el adecuado desempeño económico.

\subsection{Reglas: formales e informales}

En la conceptualización que la NEI les otorga a las reglas dentro de un determinado sistema institucional se distingue entre informales y formales, de esta manera, North (1994) define a las informales, como aquellas que nunca han sido diseñadas conscientemente y que las personas se interesan en mantener, (North, 1990) enumera tres tipos de restricciones informales, que surgen para coordinar la coordinación humana repetida: i) Las extensiones, elaboraciones y modificaciones de las reglas formales; ii) Las normas de comportamiento sancionadas socialmente; y iii) Los patrones de conducta aplicados internamente. En un ambiente de información incompleta, permiten reducir los costos de interacción, al delimitar el espacio de acción y de reacción ante cada eventualidad.

María Leonela Artavia Jiménez, Shirley Benavides Vindas y Álvaro Martín Parada Gómez 
Las reglas formales, por su parte, incluyen las restricciones de tipo económico (que definen los derechos de propiedad), político (que establecen la estructura básica de decisión y control jerárquica de la forma de gobierno y su programa de gobierno), y los contratos (que contienen las estipulaciones específicas de un acuerdo particular de intercambio). La función principal de estas reglas será la de facilitar el intercambio y, con ello, reducir los costos de transacción, conceptualización que se abarcará en el apartado 9.

Esas reglas y normas a seguir orientan el desempeño de los sectores productivos, los cuales, desde la perspectiva del enfoque de la burocracia como poder, se convierten en un mecanismo para ejercerlo, que se caracteriza por la obediencia, basado en lo racional y legal, en la normativa y jerarquía de la institucionalidad. Asimismo, en este tema es posible encontrar diversas teorías organizacionales, tales como la del comportamiento, la de las nuevas relaciones humanas, organización tradicional, entre otras. Pese a ello, los diversos enfoques son consistentes con la importancia de las instituciones y su efecto en la dinámica de las sociedades a las que pertenecen, como mecanismos de poder.

\section{El Estado}

Para la NEl, el papel del Estado es el de ser un ente facilitador de derechos de propiedad debidamente establecidos, de manera que se propicie el mayor bienestar social posible. Se considera esencial su existencia para el crecimiento económico, aunque paradójicamente tiende a ser una de las principales fuentes de declinación económica.

Haciendo uso de un modelo simple de Estado, North (1981) explica la tendencia a otorgar derechos de propiedad ineficientes, lo que impide el crecimiento sostenido de las economías, además de la inestabilidad de los estados, que conducen a la declinación económica. El modelo utilizado por North (1981) tiene tres características esenciales:

i. El Estado intercambia servicios básicos-protección y justicia- por rentas. Este intercambio tiene un doble objetivo; en primer lugar, especificar las reglas de juego que establecen las formas de competir y cooperar en sociedad y, segundo, reducir los costos de transacción. Hay economías de escala en la provisión de estos servicios especializados.

ii. El Estado actúa como un monopolista que discrimina a los contribuyentes y diseña derechos de propiedad diferentes para cada grupo, con el propósito de incrementar sus rentas al máximo posible.

iii. El Estado está restringido por los costos de oportunidad de los contribuyentes, ya que existen rivales potenciales para proporcionar este tipo de servicios. Los rivales son otros

94

María Leonela Artavia Jiménez, Shirley Benavides Vindas y Álvaro Martín Parada Gómez

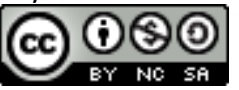

Revista Economía y Sociedad by Universidad Nacional is licensed under a CreativeCommons Reconocimiento-NoComercial- 
Estados o gobernantes potenciales del mismo Estado. El poder de monopolio del gobernante es una función de la oferta de sustitutos para varios grupos de contribuyentes.

Por último, al igual que el Estado impacta el desempeño económico, situaciones exógenas, de declive e inestabilidad también lo afectan, de manera que están en un constante proceso de retroalimentación, y este proceso es regulado a través de las reglas formales e informales. De ahí la importancia de tomar en cuenta y dar seguimiento a los estudios relacionados con el desempeño del Estado y, por lo tanto, el de sus instituciones como actores centrales en el desarrollo. Esto debido a la relación de retroalimentación entre los diversos sectores, públicos y privados, lo que constituye un elemento importante para dinamizar el crecimiento económico y el bienestar social en los diversos territorios. Además, es un referente importante para el análisis de la economía política comparada, en términos de la relación de las dinámicas económicas, políticas y sociales, como un conjunto.

Tanto la relación, Estado-empresarios como la de Estado-sociedad, son vitales para lograr un desarrollo equitativo que genere bienestar. Por supuesto, estas alianzas estratégicas demuestran que los Estados se tornan complejos, donde la burocracia debe ser eficiente $y$ racional para dinamizar, robustecer y regular los mercados, necesarios para el desarrollo exitoso de los países.

El valor agregado de la relación estratégica Estado-empresa es básico para ser un generador de procesos sociales y económicos, los cuales permea de manera directa al estimular los sistemas de producción de una forma dinámica y novedosa. Desde esta perspectiva, el Estado tiene una injerencia importante -aunque no única- en la organización empresarial de los territorios y, por lo tanto, en el desenvolvimiento de los empresarios.

\section{Costos de transacción}

Otro elemento importantísimo que trata la NEI es el de costos de transacción. En este sentido, la teoría neoclásica desarrolla su modelo sin tomar en cuenta que todo intercambio significa un costo que necesariamente afecta el desempeño económico.

Existen costos de diverso tipo relacionados con el acceso a la información, coordinación, negociación y organización; así se presentan los de transacción entre los diferentes actores de la sociedad, y para ello se debe tener información transparente sobre el comportamiento de los mercados. Entre estos aspectos se requiere saber de los precios y ejecución de contratos, por los riesgos que implican a los agentes que intervienen en el intercambio. Es parte de la base teórica de la corriente neoinstitucionalista: los costos de transacción, que son elementales para las decisiones de los diversos agentes económicos, en los cuales la empresa se origina, como

María Leonela Artavia Jiménez, Shirley Benavides Vindas y Álvaro Martín Parada Gómez 
organización y unidad económica que debe aportar a la generación y distribución del ingreso y el mejoramiento de las condiciones de la sociedad.

Según la NEI, la función de costos de producción se define como:

Donde:

$$
C T=C \operatorname{trf}+C \operatorname{trs}
$$

CT: Costos totales

$\mathrm{C}_{\text {trf: }}$ Costos de transformación

$\mathrm{C}_{\text {trs: }}$ : Costos de transacción

Por otra parte, en lo que respecta a los costos, desde la teoría institucional estos se relacionan con las transacciones, los cuales están vinculados al tipo de tecnología que se implemente y a la institucionalidad de cada sociedad. Se tiene como costos de transacción aquellos relativos a: i) especificar lo que es intercambiado y ii) hacer efectivos los acuerdos efectuados. Estos costos son, sobre todo, producto de las condiciones de incertidumbre a las que se enfrentan los agentes, así como el riesgo moral, por lo cual no deberían dejarse de lado.

En términos generales, lograr una adecuada productividad en las operaciones se asocia con una mejor relación entre los recursos asignados al proceso de producción y a la disminución de los costos unitarios en función de la capacidad instalada; además, de la absorción de costos fijos, en el desarrollo de las estrategias de producción y comercialización. Coase (1960) advertía que efectuar transacciones es costoso y, por lo tanto, las instituciones juegan un rol decisivo en la captura de las ganancias potenciales del comercio. La información asimétrica favorece el aumento de los costos de operación, razón por la cual las instituciones deben generar políticas que eviten la generación de esas asimetrías para la toma de decisiones empresariales y así contribuir a un equilibrio estratégico entre esos actores, para que cuenten con la transparencia necesaria y racional como apoyo exógeno sustantivo al sector productivo en general.

La EIO, mediante Commons (1931), indicó que la transacción como unidad básica del análisis económico y distinguió tres tipos: de negociación, de administración y de racionamiento (Parada, 2003). Sin embargo, es en la NEI donde se encuentra un mayor desarrollo de la teoría de costos de transacción, mientras que para la EIO la lógica de reducción de los costos de transacción entre empresas se fundamenta, principalmente, en estrategias pecuniarias que apuntan al control de los mercados y al fortalecimiento del poder económico de las corporaciones (Knoedler, 1995). En este sentido, cobra importancia la presencia de competencia, pues genera una reducción de los costos, ya que al haber más individuos u organizaciones en el juego, estos buscarán ser más competitivos para permanecer dentro de las opciones de los consumidores.

96

María Leonela Artavia Jiménez, Shirley Benavides Vindas y Álvaro Martín Parada Gómez

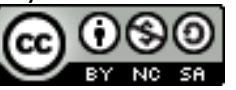

Revista Economía y Sociedad by Universidad Nacional is licensed under a CreativeCommons Reconocimiento-NoComercialCompartirlgual 4.0 Internacional License.

Creado a partir de la obra en http://www.revistas.una.ac.cr/index.php/economia 
Además, el cumplimiento de las reglas de juego delimitadas por las instituciones no siempre se da. De hecho, según North: "la aplicación u observancia de los contratos ha sido siempre el obstáculo principal para el aumento de la especialización y de la división del trabajo (1990, p. 33). Por otra parte, la NEI reconoce que el establecimiento de derechos de propiedad perfectamente especificados implica costos, así como la obtención de información, como ya se ha indicado, situación que la teoría neoclásica no incluye y que para North explica el porqué de que algunas economías no hayan sido aún capaces de mejorar sus condiciones sociales y económicas, puesto que se les aplican "recetas" que no incorporan los elementos pertinentes.

\section{Relación entre la Institucionalidad y el Desarrollo}

En el modelo neoclásico, se considera a las instituciones como variables exógenas; por lo que no deberían afectar los procesos de intercambio ni las decisiones de los agentes económicos racionales, debido a que el insumo para ellos, en una economía dada, son las variaciones de los precios relativos y la dotación de recursos; el mercado se encarga de la coordinación de las elecciones económicas, donde los costos de transacción son cero. Tampoco existen los costos de oportunidad; en el caso de la institucionalidad pública, se ubican los contratos, derechos de propiedad e información como variables dadas y en el mercado se llevan a cabo transacciones sin necesidad de dicha institucionalidad. No obstante, la teoría institucionalista ha puesto sobre la mesa la existencia de las instituciones y su rol delimitador, que permite o impide el desarrollo.

Por otra parte, como complemento a los enfoques analíticos para comprender de mejor forma los fenómenos económicos relacionados, en este caso en particular, con la dinámica empresarial, surge la concepción estatal y la burocracia. Aquí, el mercado se conceptualiza como una institución compleja, aunque es claro que no necesariamente es el mejor ni el único mecanismo utilizado para la asignación de recursos, pero se incluye en el enfoque debido a la influencia directa que tiene en las diversas instituciones para dinamizar o limitar el desenvolvimiento empresarial, desde la perspectiva de una economía dinámica, es decir, es esta la arista institución que posee el mercado, no solamente espacio en el que se intercambian bienes y servicios.

El avance del neoinstitucionalismo, se complementa con los procesos de análisis relacionados con las teorías de la regulación, la de costos de transacción, fallas de mercado y políticas públicas, la incertidumbre y la calidad de la información; todo ello debido a la necesidad humana de asociarse para el proceso productivo y de intercambio y así lograr la satisfacción de sus necesidades de todo orden (material y espiritual), por lo cual requiere de normas, incentivos y parámetros que le orienten en sus decisiones. Las instituciones, pese a sus limitaciones, afectan, igual o más que los precios, el comportamiento y las elecciones de los agentes económicos. 
Como se ha indicado, las instituciones ofrecen una estructura estable, que contribuye a disminuir la incertidumbre por parte de los individuos ante las diversas situaciones que puedan acontecer. Sin embargo, esto no quiere decir que esta sea la mejor estructura posible, sino, simplemente, que ofrece un marco a seguir por las personas ante determinada situación.

De esta manera, queda ilustrada la relación entre instituciones y progreso económico, dejando la puerta abierta para una gran cantidad de investigaciones sobre la influencia de la institucionalidad en el desarrollo productivo de las diferentes regiones costarricenses.

\section{La regulación en los bienes públicos}

El bien público, tradicionalmente, ha sido elaborado con una dotación de factores productivos como el trabajo, capital, tecnología e infraestructura pública. No obstante, este bien, en su forma aparencial, se muestra y oferta con una envestidura pública, pero en esencia es elaborado con la combinación del capital privado y público. Autores como Heritier, MuellerDebus y Thauer (2015) afirman que la intervención estatal en la generación del bien público implica asumir costos de transacción; los cuales dependerán de las reglas establecidas por el gobierno. Al no ser cumplidas, se establecerán penalidades como multas y sanciones económicas. Desde la función del Estado, las contrataciones realizadas son garantizadas por las agencias de regulación, las cuales fijan tarifas que, en buena teoría, establecen el costo del bien, intentando que tienda al costo social para la sociedad (Hovenkamp, 2010).

El proceso de globalización ha acelerado la expansión y movilidad de capital, lo cual ha permeado la generación de bienes públicos de forma mixta. Prácticamente, bienes públicos como la generación de energía eléctrica, construcción de puertos y aeropuertos son elaborados de forma conjunta entre el capital público y privado. El institucionalismo, desde la perspectiva económica, establece, según Caballero y Soto (2015), basados en North (1990), criterios de control y proceso, así como el comportamiento humano, lo cual genera características propias en la dinámica de cada institución.

La regulación en los mercados introducida por los gobiernos establece agencias reguladoras en los distintos países para garantizar la sostenibilidad del bien o servicio público al mínimo costo social y con la calidad óptima. El objetivo es definir tarifas que benefician a los consumidores, quienes pagan un precio social de acuerdo con las condiciones de cada grupo social.

\section{La regulación una forma de intervención institucional}

El institucionalismo propicia un ambiente donde las reglas y los derechos generan la actuación regulada para los individuos. Para Godard (2002), la regulación y los incentivos son mecanismos de intervención en el campo de lo económico. La regulación asume la función de regulación 98

María Leonela Artavia Jiménez, Shirley Benavides Vindas y Álvaro Martín Parada Gómez

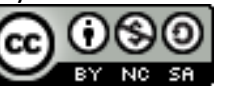

Revista Economía y Sociedad by Universidad Nacional is licensed under a CreativeCommons Reconocimiento-NoComercial- 
ante la presencia de mercados imperfectos, lo cual justifica los mecanismos de intervención del Estado. Desde la perspectiva de la teoría neoclásica, toda acción de gobierno que interfiere en el funcionamiento de los mercados se constituye en una distorsión a la eficiencia.

En la regulación eficiente, los entes de regulación impulsan el desarrollo de los modelos y prácticas de regulación que resguardan el mínimo costo directo e indirecto y la calidad exigidos a los prestadores de servicios públicos. El principio de producir, donde el costo marginal igual ingreso marginal y al precio, conlleva a óptimos de eficiencia (Varian 2010, Hirshleifer y Glazer 1992). Para Égert (2016), la regulación se relaciona con la productividad, indicando que la institucionalidad ayuda a explicar el comportamiento del factor de productividad en los países. Desde el punto de la intervención en los mercados, los resultados no están garantizados, pero existe alta probabilidad de alcanzar mayor eficiencia en el uso de los recursos productivos.

La relación de los agentes económicos, desde la lógica de la regulación fomenta el diálogo y participación en la definición tarifaria (Godard, 2002). Por lo tanto, los entes de regulación fomentan la comunicación de información permanente y la participación en los procesos de regulación por parte de los diferentes actores involucrados en el marco de independencia de criterio y de transparencia que debe caracterizar al órgano regulador. Esto es así porque los mercados de bienes regulados en países en desarrollo son imperfectos o incompletos porque persiste la información imperfecta.

La información de los mercados es atomizada y asimétrica entre los agentes económicos. En términos microeconómicos se cumple la "elección adversa"; es decir, hay acciones que ocultan información de parte de agentes en contra de consumidores, en términos de calidad de los bienes (Varian 2010). Desde el punto de vista de la intervención pública, las oficinas de la competencia intentan resguardar los derechos de los consumidores; no obstante, las acciones son insuficientes para resguardar el bienestar. Ante esto, el Estado interviene con mecanismos típicamente utilizados que se introducen a través de la política pública. Chetty (2015) afirma que algunos aspectos psicológicos intervienen en la definición de la política cuando se establecen tarifas, incentivos e impuestos; es decir, aspectos esenciales de la regulación. También argumenta que se generan efectos relacionados que pueden ser medidos econométricamente $y$, finalmente, los hacedores de política toman decisiones en el bienestar.

\section{Conclusiones}

a) En el marco del análisis, al momento de valorar la eficiencia del nivel meso, se encuentran las características del territorio, las cuales son particularmente diferentes a los centros de desarrollo y, por lo tanto, las instituciones -públicas y privadas- deben ajustarse a las condiciones asimétricas que presentan, de acuerdo con sus limitaciones o potencialidades.

María Leonela Artavia Jiménez, Shirley Benavides Vindas y Álvaro Martín Parada Gómez 
b) Las Instituciones delimitan y organizan la interacción humana. Estas reglas de juego influyen necesariamente en la evolución del desempeño de las economías y cumplen un rol de reducción de la incertidumbre, pues ofrecen una estructura estable.

c) Un mayor desarrollo institucional -instituciones fuertes- son fundamentales para el buen desenvolvimiento de los sectores productivos en un país, apoyando temas estratégicos tales como: procesos de innovación y de conocimiento y aprensión de las nuevas tecnologías desarrolladas, que generan mejores condiciones para la producción y comercialización del parque empresarial de los diversos territorios.

d) Las redes institucionales forman un pilar relevante para el buen desempeño de las empresas, debido a que generan o limitan las condiciones para lograr una adecuada competitividad de los sectores productivos y del país en términos generales; aspectos que implican una mejor interacción entre la oferta y demanda del mercado laboral.

e) Todos los esfuerzos institucionales, e incluso organizacionales, deberían generar una articulación estratégica, que posibilite el acceso oportuno a las fuentes de conocimiento y de innovación, así como de experiencias para el desarrollo productivo, no solo dirigido al actual y consolidado parque empresarial, sino también al incipiente, como lo son los emprendimientos estimulados por procesos de formación e incubación que ofrece un país o región.

f) La información asimétrica, favorece el aumento de los costos de operación, razón por la cual, las instituciones deben generar políticas que eviten que dicho comportamiento se torne en asimetrías para la toma de decisiones empresariales y así contribuir a un equilibrio estratégico entre esos actores y los sectores productivos.

g) La economía institucional ofrece un marco más amplio de análisis en comparación con la propuesta tradicional de la economía neoclásica porque integra el proceso económico, social, ambiental y cultural como parte del sistema económico. Desde la perspectiva del desarrollo productivo, esas relaciones son complejas y los precios explican solo parcialmente la dinámica de los agentes económicos.

h) Una de las rutas para el fortalecimiento de los sectores productivos es un mayor desarrollo institucional dirigido en dos líneas, la primera hacia el fomento del desarrollo integral de las personas, que son quienes conforman la sociedad e integran la fuerza laboral, y la segunda, hacia la promoción de dinámicas innovadoras que incluyan la implementación de nuevas tecnologías.

\section{Referencias}

Caballero, G \& Soto, D. (2015). The Diversity and Rapprochement of Theories of Institutional Change: Original Institutionalism and New Institutional Economics. Journal of $\begin{array}{lll}\text { economics } \quad \text { 4(49), } & \text { 947-977. }\end{array}$ http://dx.doi.org/10.1080/00213624.2015.1105021

100

María Leonela Artavia Jiménez, Shirley Benavides Vindas y Álvaro Martín Parada Gómez

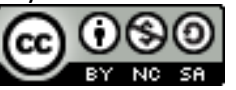

Revista Economía y Sociedad by Universidad Nacional is licensed under a CreativeCommons Reconocimiento-NoComercial- 
Caselet, M. (2004). Construcción institucional del mercado en la economía del conocimiento. Economía UNAM, 2(1).52-63, Recuperado de: http://www.scielo.org.mx/scielo.php?pid=S1665952X2004000200005\&script=sci_abstract

Chetty, R. (2015). Behavioral Economics and Public Policy: A Pragmatic Perspective. American Economic Review: Papers \& Proceedings, 5(105), 1-33. Doi: https://doi.org/10.1257/aer.p20151108

Coase, R., H. (1960). The problem of social cost. Journal of Law and Economics, 3, 1-44. Recuperado de http://www2.econ.iastate.edu/classes/tsc220/hallam/Coase.pdf

Commons, J., R. (1931). Institutional Economics. American Economic Review: History of Economic Thought Articles, (21), 648-657. Recuperado de http://la.utexas.edu/users/hcleaver/368/368commonsoninstitutionalecontable.pdf

Comisión para la Promoción de la Competencia (2017). Comisión para promover la Competencia: ¿Quiénes somos? Recuperado de https://www.coprocom.go.cr/

Égert B. (2016). Regulation, Institutions, and Productivity: New Macroeconomic Evidence from OECD Countries. American Economic Review: Papers \& Proceedings, 106 (5), 109 113. Doi: http://dx.doi.org/10.1257/aer.p20161026

Esser, K., Hillebrand, W., Messner, D., \& Meyer-Stamer, J. (1996). Competitividad Sistémica: Nuevo Desafío para las Empresas y la Política. CEPAL Review, 59, 11-38. Recuperado de

https://repositorio.cepal.org/bitstream/handle/11362/10568/59039053l.pdf?seque $\underline{\text { nce }=1}$

Godard, J. (2002). Institutional Environments, Employer Practices, and States in Liberal Market Economies. Industrial Revolutions: A Journal of economy and society, 2(41), 249-286. Doi: https://doi.org/10.1111/1468-232X.00245

Heritier, A., Mueller-Debus, A., \& Thauer, C. (2009) The Firm as an Inspector: Private Ordering and Political Rules. Recuperado de https://www.cambridge.org/core/journals/business-and-politics/article/firm-as-aninspector-private-ordering-and-politicalrules/503B58B82C021C5E198455BBC933E72F 
Hovenkamp, H. (2010). Coasean markets. Published online Springer Science Business Media. European Journal of Law and Economics, 1(31), 63-90. Doi: https://doi.org/10.1007/s10657-010-9197-4

Hirshleifer, J. \& Glazer, A. (1992). Microeconomía, Teoría y Aplicaciones. 5ta ed. Distrito Federal, México: Prentice Hall.

Knoedler, J., T. (1995). "Transaction Cost Theories of Business Enterprise from Williamson and Veblen: Convergence, Divergence, and Some Evidence". Journal of Economic Issues, 2(29), 2-29. Doi: https://doi.org/10.1080/00213624.1995.11505675

Ministerio de Economía, Industria y Comercio de Costa Rica. (2017). MEIC: Mejora regulatoria. Recuperado de https://www.meic.go.cr/meic/web/42/mejora-regulatoria.php

North, D., C. (1981). Structure and Change in Economic History. Politics \& Society, 4(11), 511512.

DOI: https://books.google.co.cr/books/about/Structure and Change in Economic Histo ry.html?id= WDAQgAACAAJ\&redir esc=y

North, D., C. (1990). Instituciones, Cambio Institucional y Desempeño Económico. Chile: Fondo de Cultura Económica. Recuperado de https://es.scribd.com/doc/212845951/Douglass-North-Instituciones-CambioInstitucional-y-Desempeno-Economico-parte-Primera

North, D., C. (1994). Economic Performance Through Time. The American Economic Review, 3(84), 359-368. Recuperado de http://www.jstor.org/stable/2118057

Parada, J., J. (2003). Economía institucional original y nueva economía institucional: semejanzas y diferencias. Revista de Economía Institucional, 8(5), 92-116. Recuperado de https://revistas.uexternado.edu.co/index.php/ecoins/article/view/206

Robles, G. A. (1998). El Pensamiento Económico de Douglass C. North. Revista de la Facultad de Ciencias Económicas: Laissez Faire, 9, 13-32. Recuperado de http://www.eumed.net/cursecon/textos/Prado North.pdf

Varian, H., R. (2010). Un enfoque actual Microeconomía Intermedia (9na ed.). Barcelona, España: Antoni Bosch.

102

María Leonela Artavia Jiménez, Shirley Benavides Vindas y Álvaro Martín Parada Gómez

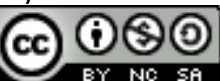

Revista Economía y Sociedad by Universidad Nacional is licensed under a CreativeCommons Reconocimiento-NoComercialCompartirlgual 4.0 Internacional License.

Creado a partir de la obra en http://www.revistas.una.ac.cr/index.php/economia 